\title{
Enhanced settling of nonheavy inertial particles in homogeneous isotropic turbulence: The role of the pressure gradient and the Basset history force
}

Citation for published version (APA):

van Hinsberg, M. A. T., Clercx, H. J. H., \& Toschi, F. (2017). Enhanced settling of nonheavy inertial particles in homogeneous isotropic turbulence: The role of the pressure gradient and the Basset history force. Physical Review E, 95(2), 1-8. [023106]. https://doi.org/10.1103/PhysRevE.95.023106

DOI:

10.1103/PhysRevE.95.023106

Document status and date:

Published: 09/02/2017

Document Version:

Publisher's PDF, also known as Version of Record (includes final page, issue and volume numbers)

\section{Please check the document version of this publication:}

- A submitted manuscript is the version of the article upon submission and before peer-review. There can be important differences between the submitted version and the official published version of record. People interested in the research are advised to contact the author for the final version of the publication, or visit the DOI to the publisher's website.

- The final author version and the galley proof are versions of the publication after peer review.

- The final published version features the final layout of the paper including the volume, issue and page numbers.

Link to publication

\footnotetext{
General rights

- You may freely distribute the URL identifying the publication in the public portal. follow below link for the End User Agreement:

www.tue.nl/taverne

\section{Take down policy}

If you believe that this document breaches copyright please contact us at:

openaccess@tue.nl

providing details and we will investigate your claim.
}

Copyright and moral rights for the publications made accessible in the public portal are retained by the authors and/or other copyright owners and it is a condition of accessing publications that users recognise and abide by the legal requirements associated with these rights.

- Users may download and print one copy of any publication from the public portal for the purpose of private study or research.

- You may not further distribute the material or use it for any profit-making activity or commercial gain

If the publication is distributed under the terms of Article 25fa of the Dutch Copyright Act, indicated by the "Taverne" license above, please 


\title{
Enhanced settling of nonheavy inertial particles in homogeneous isotropic turbulence: The role of the pressure gradient and the Basset history force
}

\author{
M. A. T. van Hinsberg, ${ }^{1}$ H. J. H. Clercx, ${ }^{1}$ and F. Toschi ${ }^{1,2}$ \\ ${ }^{1}$ Fluid Dynamics Laboratory and J.M. Burgers Centre for Fluid Dynamics, Department of Physics, Eindhoven University of Technology, \\ P.O. Box 513, 5600MB Eindhoven, The Netherlands \\ ${ }^{2}$ Department of Mathematics and Computing Science, Eindhoven University of Technology, P.O. Box 513, \\ 5600MB Eindhoven, The Netherlands \\ (Received 4 November 2016; published 9 February 2017)
}

\begin{abstract}
The Stokes drag force and the gravity force are usually sufficient to describe the behavior of sub-Kolmogorovsize (or pointlike) heavy particles in turbulence, in particular when the particle-to-fluid density ratio $\rho_{p} / \rho_{f} \gtrsim 10^{3}$ (with $\rho_{p}$ and $\rho_{f}$ the particle and fluid density, respectively). This is, in general, not the case for smaller particle-to-fluid density ratios, in particular not for $\rho_{p} / \rho_{f} \lesssim 10^{2}$. In that case the pressure gradient force, added mass effects, and the Basset history force also play important roles. In this study we focus on the understanding of the role of these additional forces, all of hydrodynamic origin, in the settling of particles in turbulence. In order to qualitatively elucidate the complex dynamics of such particles in homogeneous isotropic turbulence, we first focus on the case of settling of such particles in the flow field of a single vortex. After having explored this simplified case we extend our analysis to homogeneous isotropic turbulence. In general, we found that the pressure gradient force leads to a decrease in the settling velocity. This can be qualitatively understood by the fact that this force prevents the particles from sweeping out of vortices, a mechanism known as preferential sweeping which causes enhanced settling. Additionally, we found that the Basset history force can both increase and decrease the enhanced settling, depending on the particle Stokes number. Finally, the role of the nonlinear Stokes drag has been explored, confirming that it affects settling of inertial particles in turbulence, but only in a limited way for the parameter settings used in this investigation.
\end{abstract}

DOI: 10.1103/PhysRevE.95.023106

\section{INTRODUCTION}

Settling of particles occurs in many industrial and environmental systems. Industrial examples range from spray painting, combustion engines, and wastewater treatment; see, for example, Refs. [1,2]. Environmental examples are raindrops in clouds, sedimentation, resuspension and transport of sand particles in rivers and estuaries, and plankton transport in oceans. In these systems turbulence influences the particle dynamics [3]. For example, it is well known that homogeneous isotropic turbulence can enhance the settling velocity of particles, as first shown by Wang and Maxey [4]. Many studies [5-8] have been done, both numerically and experimentally, to investigate this phenomenon in more detail. The mechanism proposed for explaining enhanced settling is the preferential sweeping effect [4]; see the schematic picture in Fig. 1. Suppose a heavy inertial particle enters the vortex with clockwise circulation on top. This particle will preferentially move clockwise while being spun out of the vortex center. Due to the sweeping effect it will leave the vortex, on average, at the downward-moving side of it. A similar conclusion can be drawn when the inertial particle enters a vortex with counterclockwise circulation. Summarizing, particles are swept out of the vortices and, with the help of gravity, tend to end up at the downward-moving side of the vortices, thus promoting enhanced settling.

Most numerical studies on the behavior of (point) particles in turbulence have only included the Stokes drag force and the gravity force in the equations of motion describing the particle dynamics and evaluating particle trajectories. This combination of forces is considered adequate when studying the behavior of particles or droplets with a particle-to-fluid density ratio $\rho_{p} / \rho_{f}$, with $\rho_{f}$ the fluid density and $\rho_{p}$ the particle density, above about 1000 (typical for droplets and solid particles in air). On the other hand, for particles with density ratios $\rho_{p} / \rho_{f} \lesssim O(100)$ (which we call nonheavy particles for convenience), this combination of forces is not sufficient for a proper evaluation of particle trajectories in turbulent flows [9-11]. For the case of these nonheavy particles, more terms in the full Maxey-Riley equation need to be retained [12]. The full Maxey-Riley equation includes, for example, the pressure gradient force, added mass effects, and the Basset history force. The implementation of these forces makes the calculation more complex and computationally more expensive. Advances in hardware computing power and algorithm efficiency make the additional computational cost associated with the evaluation of these forces (and particle trajectories derived from them) more acceptable compared to a decade ago [13-15]. Another subtlety is related with the particle size compared to the Kolmogorov length scale $\eta$ (or the dissipation scale) of the turbulent flow. When the particle radius becomes of the order of or bigger than $8 \eta$ even the Maxey-Riley equation (with Faxén correction [16-18]) is not sufficient anymore; see also Ref. [19]. In this case one needs, in principle, to conduct fully resolved simulations where both the shape of the particle and the boundary layer around the particle are fully resolved. A few recent examples concern the settling of finite-size particles in homogeneous isotropic turbulence by means of interface-resolved simulations [20,21]. Although this is, in principle, an accurate approach for this purpose, it is computationally much more expensive than the point-particle approach based on the Maxey-Riley equation. Also the number of particles that can be simulated is greatly reduced when conducting fully resolved simulations. In our approach this will not be the case because we assume that the 


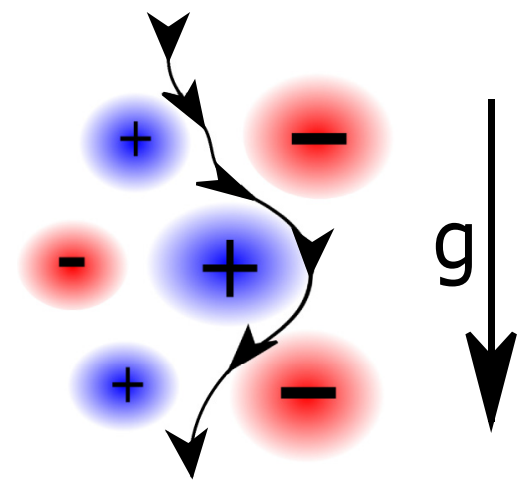

FIG. 1. Schematic picture of the trajectory of a settling particle in homogeneous and isotropic turbulence. The blue and red circles represent, schematically, vortices with clockwise $(+)$ and counterclockwise (-) circulation. Due to preferential sweeping, particles can obtain an enhanced settling velocity by preferentially selecting flow regions with downward velocities.

particle radius $a$ is sufficiently small, i.e., $a \lesssim \eta$. Therefore, a large number of particles can easily be simulated (typically in the order of $10^{6}$ to $10^{7}$ ). This is useful in order to explore a large range of particle properties, yet obtaining enough statistics for each particle type.

The paper is organized as follows. First, we start by introducing the equations of motion for both the fluid and the inertial particles. Second, we investigate a simplified system where inertial particles are released and subsequently settle in a two-dimensional flow, which consists of one basic vortex. This represents a sort of "toy model" useful to elucidate the main influences of the different hydrodynamic forces on settling and serves as a building block for the full system consisting of settling inertial particles in turbulence. Subsequently, we discuss the influence of the Basset history force and of the pressure gradient force, in the case of homogeneous isotropic turbulence. Finally, we consider briefly the effect of the nonlinear drag force, and we investigate how nonlinear drag modifies (enhanced) settling of nonheavy inertial particles. We conclude this paper with a brief summary of the main results.

\section{EQUATIONS OF MOTION FOR FLUID AND INERTIAL PARTICLES}

We assume a very dilute dispersed phase with a small total particle volume fraction $\phi$, typically $\phi \lesssim 10^{-3}$. The particle size is assumed to be small, i.e., smaller than the smallest length scales in the flow, which, in the case of homogeneous isotropic turbulence, is the Kolmogorov length scale $\eta$. Under these conditions we can use one-way coupling [22]. The particles are only influenced by the fluid, but they do not disturb the fluid flow itself and do not interact (hydrodynamically or otherwise) with other particles as well. For the simulation of the flow we use an Eulerian approach. The flow is computed using a standard pseudospectral code solving the incompressible Navier-Stokes equations; see Ref. [23] for some details. The pseudospectral code is fully based on dimensionless variables. The computational box is $(2 \pi)^{3}$, and periodic boundary conditions are applied. The flow is forced by injecting (dimensionless) energy on the largest scales (smallest wave numbers). The forcing algorithm inserts energy at a constant rate; see also Lamorgese et al. [24].

For the particle phase we use a Lagrangian approach. Particle trajectories in a Lagrangian frame of reference satisfy

$$
\frac{d \mathbf{x}_{p}(t)}{d t}=\mathbf{u}_{p}(t)
$$

with $\mathbf{x}_{p}(t)$ the particle position and $\mathbf{u}_{p}(t)$ its velocity. According to Maxey and Riley [12], the equation of motion for an isolated rigid spherical particle in a nonuniform velocity field $\mathbf{u}(\mathbf{x}, t)$ is given by

$$
\begin{aligned}
m_{p} \frac{d \mathbf{u}_{p}}{d t}= & 6 \pi a \mu\left(\mathbf{u}-\mathbf{u}_{p}\right)+m_{f} \frac{D \mathbf{u}}{D t}-\left(m_{p}-m_{f}\right) g \mathbf{e}_{z} \\
& +\frac{1}{2} m_{f}\left(\frac{D \mathbf{u}}{D t}-\frac{d \mathbf{u}_{p}}{d t}\right) \\
& +3 \sqrt{3 \mu a m_{f}} \int_{-\infty}^{t} \frac{d \mathbf{u}(\tau) / d \tau-d \mathbf{u}_{p}(\tau) / d \tau}{\sqrt{t-\tau}} d \tau \\
= & \mathbf{F}_{\mathrm{St}}+\mathbf{F}_{\mathrm{P}}+\mathbf{F}_{\mathrm{G}}+\mathbf{F}_{\mathrm{AM}}+\mathbf{F}_{\mathrm{B}} .
\end{aligned}
$$

The equation of motion includes time derivatives of the form $d / d t$ taken along the particle path and the derivatives of the form $D / D t$ taken along the path of a fluid element. The particle mass is given by $m_{p} ; a$ is the radius of the particle; $\mu=$ $\rho_{f} v$ is the dynamic viscosity, with $\rho_{f}$ and $v$ the density of the fluid and its kinematic viscosity, respectively; $m_{f}$ is the mass of the fluid element with a volume equal to that of the particle; and $\mathbf{e}_{z}$ is the unit vector in the opposite direction of the gravitational force. The forces on the right-hand side of this equation denote, respectively, the Stokes drag force, the local pressure gradient force (over the particle diameter) in the undisturbed fluid, the gravitational force, the added mass contribution, and the Basset history force. For the latter, see Refs. [25,26]. Although we stick here to the traditional kernel derived by Basset [25] it should be mentioned that several other kernels, which are found to depend on the type of flow, are proposed in the literature; see, for example, the overviews in Refs. [27,28].

Equation (2) is valid when $a \ll \eta$, although adding the Faxén correction [16-18] will relax this condition somewhat; see Sec. I. As in this study we did not consider Faxén corrections, we will limit ourselves to small $a$. Furthermore, the particle Reynolds number must also be small $\left(\operatorname{Re}_{p} \ll 1\right)$ to assume validity of the Maxey-Riley equation [12]. When $\operatorname{Re}_{p}$ becomes larger nonlinear effects are going to play a role, like nonlinear Stokes drag, and lift forces. We will briefly discuss the effects of the nonlinear drag in Sec. V.

We rewrite Eq. (2) as follows:

$$
\begin{aligned}
\frac{d \mathbf{u}_{p}}{d t}= & \frac{1}{\tau_{p}}\left(\mathbf{u}-\mathbf{u}_{p}\right)+\frac{1}{R_{\rho}} \frac{D \mathbf{u}}{D t}-\left(1-\frac{1}{R_{\rho}}\right) g \mathbf{e}_{z} \\
& +\frac{1}{2 R_{\rho}}\left(\frac{D \mathbf{u}}{D t}-\frac{d \mathbf{u}_{p}}{d t}\right) \\
& +\frac{3}{\sqrt{2 \pi \tau_{p} R_{\rho}}} \int_{-\infty}^{t} \frac{d \mathbf{u}(\tau) / d \tau-d \mathbf{u}_{p}(\tau) / d \tau}{\sqrt{t-\tau}} d \tau .
\end{aligned}
$$


TABLE I. Conversion between density ratio $R_{\rho}$ and $\beta$; see also Eq. (6). The list includes the three standard cases where the particle density is much higher than the fluid density, where they are of similar order, and where the fluid density is much higher than the one of the particles.

\begin{tabular}{lcccccccc}
\hline \hline$R_{\rho}$ & $\infty$ & 1000 & 100 & 10 & 2 & 1.2 & 1 & 0 \\
$\beta$ & 0 & 0.0015 & 0.0149 & 0.1429 & 0.6 & 0.8824 & 1 & 3 \\
\hline \hline
\end{tabular}

Here, the two remaining parameters in the Maxey-Riley equation are the particle response time $\tau_{p}=\frac{m_{p}}{6 \pi a \mu}=\frac{2 v}{9 a^{2}} \frac{\rho_{p}}{\rho_{f}}$ and the particle-to-fluid density ratio $R_{\rho}=\frac{\rho_{p}}{\rho_{f}}$. This equation can be rewritten by splitting up the added mass term,

$$
\begin{aligned}
\frac{d \mathbf{u}_{p}}{d t}= & \frac{\mathbf{u}-\mathbf{u}_{p}}{\tau_{p}^{*}}+\beta \frac{D \mathbf{u}}{D t}-(1-\beta) g \mathbf{e}_{z} \\
& +\sqrt{\frac{3 \beta}{\pi \tau_{p}^{*}}} \int_{-\infty}^{t} \frac{d \mathbf{u}(\tau) / d \tau-d \mathbf{u}_{p}(\tau) / d \tau}{\sqrt{t-\tau}} d \tau \\
= & \mathbf{F}_{\mathrm{St}}^{*}+\mathbf{F}_{\mathrm{P}}^{*}+\mathbf{F}_{\mathrm{G}}^{*}+\mathbf{F}_{\mathrm{B}}^{*},
\end{aligned}
$$

where

$$
\begin{gathered}
\tau_{p}^{*}=\left(1+\frac{1}{2 R_{\rho}}\right) \tau_{p}=\frac{3}{3-\beta} \tau_{p}, \\
\beta=\frac{3}{2 R_{\rho}+1} .
\end{gathered}
$$

The relation between $\beta$ and $R_{\rho}$ is also shown in Table I. If the particle density is much higher than the fluid density, thus for the limiting situation $R_{\rho} \rightarrow \infty$, Eq. (4) simplifies to the classical expression for heavy particles, with only the Stokes drag force and the gravity force remaining on the right-hand side of Eq. (4). In this case, $\tau_{p}^{*}=\tau_{p}$ and $\beta=0$. Decreasing the density ratio the first term that starts to compete with the Stokes drag force and the gravity force is the Basset history force, as this force scales with $\sqrt{\beta}$. The importance of the Basset history force for inertial particles in homogeneous isotropic turbulence has already been shown by several studies [10,11,29-31]. When decreasing the density ratio $R_{\rho}$ even further, the pressure gradient force, which scales linearly with $\beta$, also becomes important. Under these circumstances also the relative importance of the Stokes drag force diminishes as the effective relaxation time $\tau_{p}^{*}$ increases.

In order to get accurate statistics in an efficient way the right choice of the computational methods is crucial. To calculate forces like the added mass and the Basset history force, also the material derivative needs to be calculated, which calls for highly accurate interpolation methods. The fluid velocity at the particle center is efficiently calculated by using a sixth-order B-spline interpolation method. This provides high accuracy for both the interpolated quantity as well as for its derivatives [32-34]. Next, the material derivative is calculated with the method described by Van Hinsberg et al. [32]. The calculation of the Basset history force is very time consuming, because the total history of the particle trajectory needs to be considered. This problem is addressed by using an exponential function for the tail of the Basset history force [13]. In this way only a limited number of time steps need to be taken into account, reducing the total computation time for the evaluation of this force by more than an order of magnitude. Improving the accuracy of the numerical calculation of the Basset history force has been an active field of research in the last decade $[13,14,35,36]$. A recent review paper [15] concludes that the method by Van Hinsberg et al. [13] gives the best balance between computing time and accuracy.

The combination of these methods allows us to calculate high amounts of particle trajectories with high accuracy and efficiency. The high number of particles (typically in the order of millions) are needed in order to cover the parameter range of interest and to obtain well-converged statistics.

\section{INERTIAL PARTICLES SETTLING THROUGH A VORTICAL FLOW}

In order to understand the influence of the different forces on the settling of inertial particles in homogeneous isotropic turbulence, we start by investigating a simple two-dimensional system. A sketch of this system is displayed in Fig. 2. In this system we consider a single vortex, centered at position $(x, y)=(0,0)$, with inertial particles released at $y=L$ (thus with a minimum distance $L$ to the vortex centre) which settle due to gravity. Many of these particles are initially homogeneously distributed in the range $-L \leqslant x \leqslant L$. The particle velocities are initialized with their terminal settling velocity in a quiescent fluid:

$$
U_{s}=\tau_{p}^{*}(1-\beta) g .
$$

When the particles reach the vertical location $y=-3 L$, thus about a distance $3 L$ below the vortex, we check for each of the particles the arrival time $t_{f}$. This time is compared with the time for particles settling with the terminal velocity and the difference between the two is denoted as the delay time
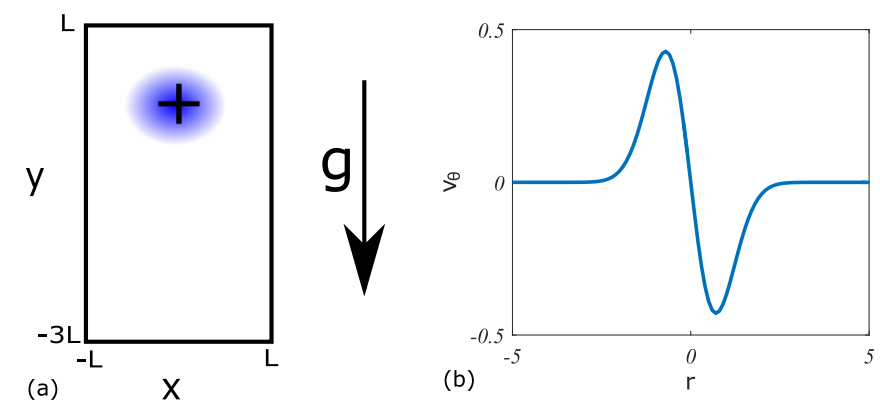

FIG. 2. Setup for the study of the influence of the different hydrodynamic forces on the settling of inertial particles in the vicinity of a single isolated vortex. (a) Schematic setup of the flow domain, where a single vortex is placed. We locate the origin of our coordinate system at the center of this vortex. The particles start with their terminal settling velocity on the top of the domain (at $y=L$ ). In order to make sure that the particles are also in a force equilibrium when leaving the domain, the domain is made longer in the $y$ direction (domain size of $L$ above and $3 L$ below the vortex). (b) Azimuthal velocity $v_{\theta}$ as a function of the distance $r$ to the center of the vortex, with $\omega_{0}=1$ and $R=1$; see Eq. (9). 
$\Delta t$, so

$$
\Delta t=t_{f}-4 L / U_{s} .
$$

By using different combinations of particle forces, we can obtain a qualitative understanding of the influence of the different forces on particle settling in the vicinity of a single vortex.

In order to have a localized vortical flow field, a shielded vortex, characterized by zero total circulation, with a smooth velocity profile is chosen. Because the vortex is shielded it must contain both positive and negative vorticity. Although there are multiple possibilities for a smooth shielded vortex, we have chosen one of the simplest cases. It is a Gaussian vortex consisting of a core with positive vorticity surrounded by a ring of negative vorticity. While the exact quantitative results on particle settling will depend on the choice of the vortex, this is not expected for the qualitative results we are interested in. The velocity field of the Gaussian vortex used in this study is given by

$$
v_{\theta}(r)=\omega_{0} r\left[\exp \left(-\frac{r^{2}}{R^{2}}\right)\right]
$$

[see Fig. 2(b)], and the vorticity field $\omega(r)$ is, according to the definition $\omega(r)=\frac{1}{r} \frac{d}{d r}\left(r v_{\theta}\right)$, given by

$$
\omega(r)=2 \omega_{0}\left(1-\frac{r^{2}}{R^{2}}\right) \exp \left(-\frac{r^{2}}{R^{2}}\right) .
$$

Here, $2 \omega_{0}$ is the maximum vorticity (at $r=0$ ) and $R$ is the typical radius (where the vorticity changes sign).

In order to fully characterize the particles in the system, we need three dimensionless parameters. We use one of the quantities $R_{\rho}$ or $\beta$ together with

$$
\begin{aligned}
\mathrm{St}^{*} & =\frac{\tau_{p}^{*}}{\tau}, \\
\mathrm{Sv}^{*} & =\frac{U_{s}}{U}=\frac{\tau_{p}^{*}(1-\beta) g}{U} .
\end{aligned}
$$

For the Stokes number $\mathrm{St}^{*}$, the particle response time $\tau_{p}^{*}$ is compared with a typical time scale $\tau$ of the flow. For the settling number $\mathrm{Sv}^{*}$ the terminal settling velocity $U_{s}$ is compared with a typical velocity of the flow $U$.

We start with investigating the case of $R_{\rho}=\infty$ or $\beta=0$. In this case $\tau_{p}^{*}=\tau_{p}$ and

$$
\begin{aligned}
& \mathrm{St}=\mathrm{St}^{*}(\beta=0)=\frac{\tau_{p}}{\tau}, \\
& \mathrm{Sv}=\mathrm{Sv}^{*}(\beta=0)=\frac{U_{s}(\beta=0)}{U}=\frac{\tau_{p} g}{U} .
\end{aligned}
$$

Furthermore, the particles are fully characterized by only two dimensionless parameters, namely St and Sv and the particle equations reduce to contain only the Stokes drag force and the gravity force. For the characteristic time and velocity scale we use $\tau=1 / \omega_{0}$ and $U=R \omega_{0}$, respectively. During the computation we use the following parameters: $\omega_{0}=1$, $R=1$, and $L=5$. As a typical example, we show in Fig. 3 the results (particle trajectories and delay time $\Delta t$ ) for $\mathrm{St}=0.5$ and $\mathrm{Sv}=0.2$. From these results we can learn that most of the trajectories result in a negative $\Delta t$, in agreement with the standard concept of enhanced settling induced by preferential
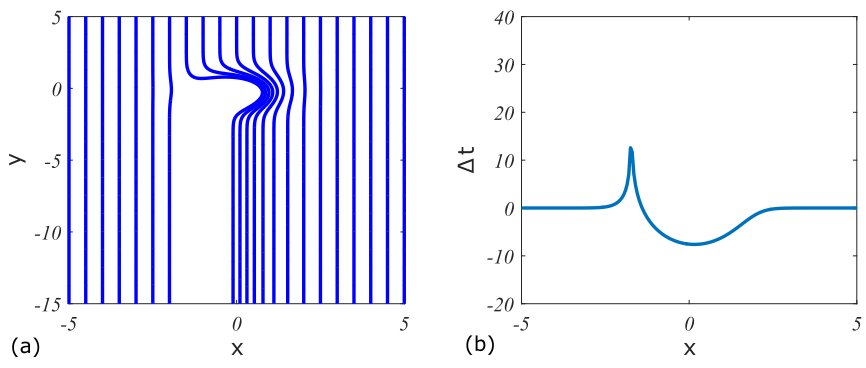

FIG. 3. Inertial particles with $\beta=0$ settling in a stationary velocity field induced by an isolated vortex with positive core vorticity; see Fig. 2. The particle parameters are $\mathrm{St}=0.5$ and $\mathrm{Sv}=0.2$. (a) Visualization of the inertial particle trajectories. (b) Delay time $\Delta t$ of the particles (plotted as a function of the starting position $x$ ) between the actual settling time compared with the time needed for the particle when it falls with the terminal settling velocity $U_{s}$; see Eq. (8).

sweeping [4]. Additionally, there is a stagnation point in the field of particle trajectories just left of and above the vortex, resulting in some particle trajectories giving a positive $\Delta t$. It is important to realize that there is no stagnation point in the flow field itself and the existence of the stagnation point in particle trajectories is caused by particle inertia. If one would average $\Delta t$ over the different particles (basically integrating the delay time $\Delta t$, shown in the right panel of Fig. 3, from $x=-L$ up to $x=L$ ), it is not immediately clear in advance if the result would be positive or negative as we have to take into account the asymptote due to the stagnation point. However, we do not start an investigation on the influence of this stagnation point as it is not relevant when comparing it to the case of homogeneous isotropic turbulence (although we should not exclude the possibility that it might have some minor statistical influence). In the case of homogeneous isotropic turbulence such a stagnation point could only survive for a limited amount of time as the flow removes such points almost immediately. Moreover, any statistical analysis would require allocation of these stagnation points in the course of time together with sufficient events of these stagnation points to acquire sufficient statistics. Therefore, we only consider a qualitative discussion of the results of settling particles affected by a single vortex, which will give meaningful insight into the effect of the different forces on particle dynamics and (enhanced) settling.

To investigate the influence of the different hydrodynamic forces, we consider an extreme case: $R_{\rho}=1.2$ with $\mathrm{St}^{*}=$ 2 and $\mathrm{Sv}^{*}=0.4$. Note that the radius of the particle is not allowed to become too large, as it should be smaller than the smallest length scale of the flow (in the single-vortex case we only have the vortex radius $R$ as a length scale). Figure 4(a) shows the particle trajectories when all the forces are included. Furthermore, the right panel in the figure shows the delay time $\Delta t$ [see Eq. (8)] for different combinations of hydrodynamic forces. From the trajectories it can be observed that some particles can end up in stable orbits around the center of the vortex. This cannot be observed when only the Stokes drag force and the gravity force are active. When adding the pressure gradient force this behavior emerges; the pressure gradient force is directed to the center of the vortex, where the pressure is lowest, thus counteracting outward motion due to inertia, 

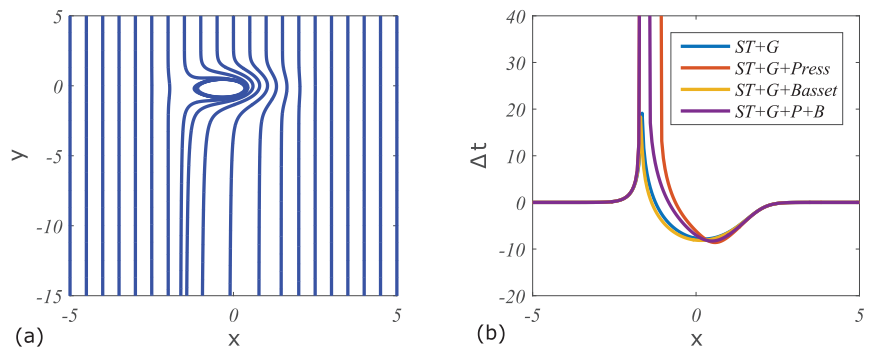

FIG. 4. Inertial particles with $R_{\rho}=1.2$ settling in a stationary velocity field [see Eq. (2)] induced by an isolated vortex with positive core vorticity. The particle parameters are $\mathrm{St}^{*}=2$ and $\mathrm{Sv}^{*}=0.4$. (a) Visualization of inertial particle trajectories. (b) The delay time $\Delta t$ as a function of the starting position $x$ when different combinations of hydrodynamic forces are included, indicated by the different colors. ST, Stokes drag force; G, gravity force; Press or P, pressure gradient force; Basset or B, Basset history force.

making a stable orbit possible. The Basset history force, on the other hand, decreases the chance of particles ending up inside the vortex, which is nicely illustrated in Fig. 4(b), where the width of the peak becomes substantially narrower when the Basset history force is included (on top of the pressure gradient force).

To conclude, we have seen that for heavy particles, with $\beta=0$, we find an enhanced settling for most of the trajectories. Increasing $\beta$ shows that this behavior can be either decreased or increased by the other forces. The pressure gradient keeps the particles longer inside the vortex (decreasing the settling velocity), while the Basset history force can counteract this behavior (thus increasing again the settling velocity). In the next section we will compare the present observations from our simplified toy model with results from the case of settling particles in homogeneous isotropic turbulence.

\section{INERTIAL PARTICLES IN HOMOGENEOUS ISOTROPIC TURBULENCE}

The most important parameter characterizing homogeneous isotropic turbulence is the Taylor-Reynolds number, defined as $\operatorname{Re}_{\lambda}=u_{r . m . s .}^{2} \sqrt{15 /(v \epsilon)}$. Here, $\epsilon$ is the dissipation rate, $u_{r . m . s .}^{2}=\frac{1}{3}\left(\left\langle u_{x}^{2}\right\rangle+\left\langle u_{y}^{2}\right\rangle+\left\langle u_{z}^{2}\right\rangle\right)$ is the mean-squared velocity of the turbulent flow, and $\langle\cdots\rangle$ denotes the spatial and time average [all flow characteristics are represented in Table II; note that all quantities are already in dimensionless form (see Sec. II)]. The (dimensionless) Kolmogorov length is $\eta=\left(v^{3} / \epsilon\right)^{1 / 4}=0.019$, which leads to $k_{\max } \eta=1.7$, ensuring sufficient resolution at small scales. Furthermore, we have put different types of particles in the flow. Each type, denoted by

TABLE II. Dimensionless flow characteristics for the DNS of homogeneous isotropic turbulence, with $u_{\text {r.m.s. }}$ the root-mean-square velocity of the turbulent flow, $v$ the kinematic viscosity, $\epsilon$ the dissipation rate, $\eta$ the Kolmogorov length scale, $\tau_{\eta}$ the Kolmogorov time scale, and $\operatorname{Re}_{\lambda}$ the Taylor-based Reynolds number.

\begin{tabular}{lccccccc}
\hline \hline Domain size & Grid points & $u_{\text {r.m.s. }}$ & $v$ & $\epsilon$ & $\eta$ & $\tau_{\eta}$ & $\operatorname{Re}_{\lambda}$ \\
\hline$(2 \pi)^{3}$ & $256^{3}$ & 1.4 & 0.005 & 1 & 0.019 & 0.072 & 103 \\
\hline \hline
\end{tabular}
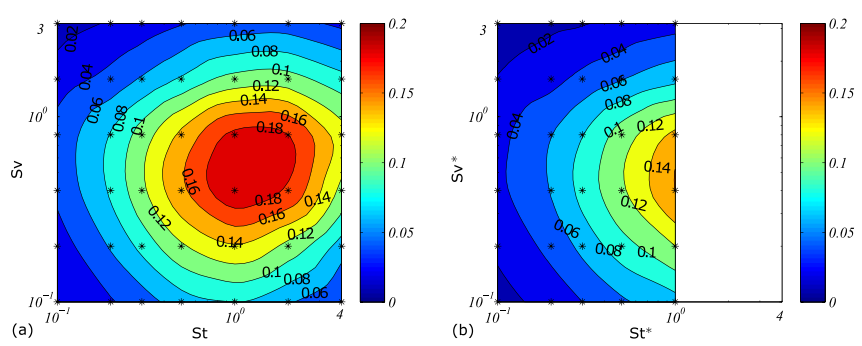

FIG. 5. The normalized enhanced settling velocity $U_{e s} / u_{\text {r.m.s. }}$ of inertial particles as a function of $\mathrm{St}^{*}$ and $\mathrm{Sv}^{*}$. Two density ratios are shown: (a) $R_{\rho}=\infty$ (i.e., $\beta=0$; for this particular case $\mathrm{St}^{*}=\mathrm{St}$ and $\mathrm{Sv}^{*}=\mathrm{Sv}$ ) and (b) $R_{\rho}=10$. For $R_{\rho}=10$ not all Stokes numbers have been simulated as the particle radius becomes bigger than the smallest length scale in the flow, as $a>\eta$ for $\mathrm{St}^{*} \gtrsim 1$.

$\mathcal{P}$, consist of 5120 inertial particles with the same physical properties. In order to get sufficient statistics, we simulated the flow with particles for 1000 large-eddy turnover times and started measuring the statistics after 100 large-eddy turnover times in order to discard the startup phase of the flow. In order to quantify $\mathrm{St}^{*}$ and $\mathrm{Sv}^{*}$ with the definitions given in Eqs. (11) and (12), respectively, we need a typical time and velocity scale. We will use $\tau_{\eta}$ and $u_{\text {r.m.s. }}$, respectively, where $\tau_{\eta}$ is the Kolmogorov time scale defined as $\tau_{\eta}=\sqrt{v / \epsilon}$.

We start again with investigating the case where $R_{\rho}=\infty$ or $\beta=0$. We measure the enhanced settling velocity $U_{e s}$, defined as

$$
U_{e s}=\left\langle-u_{p, z}\right\rangle_{\mathcal{P}}-U_{s}
$$

Here $u_{p, z}$ is the particle velocity in the $z$ direction, $U_{s}$ is defined in Eq. (7), and $\langle\cdots\rangle_{\mathcal{P}}$ denotes an average over the inertial particles belonging to one particular type $\mathcal{P}$ and over time. In Fig. 5(a) the enhanced settling velocity relative to $u_{\text {r.m.s. }}$. is shown as a function of $\mathrm{St}$ and $\mathrm{Sv}$. Around $\mathrm{St} \approx 1.5$ and $\mathrm{Sv} \approx 0.5$ we see a maximum in the enhanced settling velocity. It turns out to be approximately $20 \%$ of the root-mean-square velocity of the turbulent flow. In Fig. 6(a) we show the same data but now the relative enhanced settling velocity $U_{e s} / U_{s}$. The maximum occurs now for $\mathrm{St} \approx 1.0$ and $\mathrm{Sv} \approx 0.1$ and in terms of the terminal settling velocity in a quiescent fluid the increase is about $60 \%$.

Next we decrease the density ratio to $R_{\rho}=10$; see right panels in Figs. 5 and 6 . Note that we have to make sure that $a<\eta$; see discussion in Sec. II and Ref. [12]. Calculation of
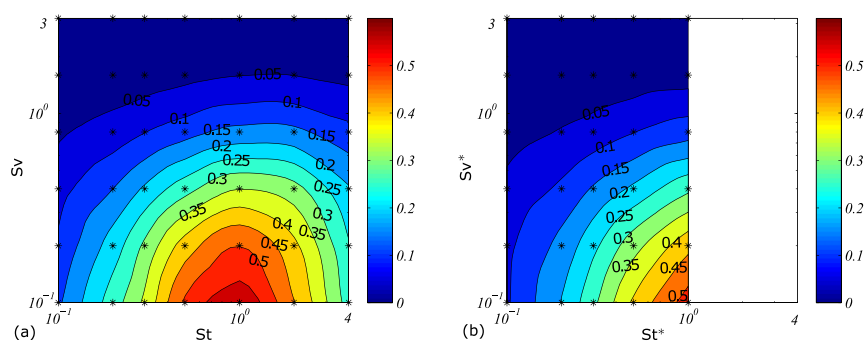

FIG. 6. The relative enhanced settling velocity $U_{e s} / U_{s}$. In this figure the same data set as in Fig. 5 has been used. 

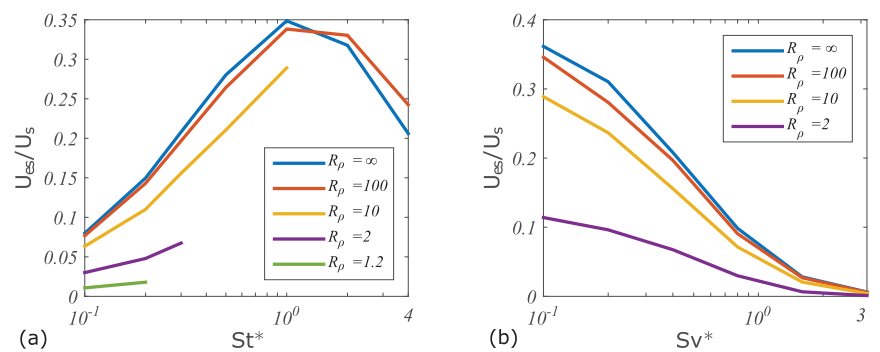

FIG. 7. Relative enhanced settling velocity for different density ratios (a) as a function of $\mathrm{St}^{*}$ with $\mathrm{Sv}^{*}=0.4$ (horizontal cut of Fig. 6) and (b) as a function of $\mathrm{Sv}^{*}$ with $\mathrm{St}^{*}=0.3$ (vertical cut of Fig. 6).

$a / \eta$ gives

$$
\frac{a}{\eta}=3 \sqrt{\frac{\mathrm{St}^{*}}{1+2 R_{\rho}}} .
$$

So, in order to maintain $a / \eta$ small enough, when decreasing $R_{\rho}$, we also need to decrease the maximum value of $\mathrm{St}^{*}$ (see Figs. 5 and 6). It should be noted that our choice for $\mathrm{St}^{*}$ turns out to be somewhat conservative (upper bounds are $\mathrm{St}^{*} \approx 2.3$ for $R_{\rho}=10, \mathrm{St}^{*} \approx 0.6$ for $R_{\rho}=2$, and $\mathrm{St}^{*} \approx 0.4$ for $R_{\rho}=1.2$ ). From Figs. 5 and 6 , one can observe that the enhanced settling velocity is somewhat decreased for $R_{\rho}=10$. The location of the maximum in $\left(\mathrm{St}^{*}, \mathrm{~Sv}^{*}\right)$ parameter space is similar as for $R_{\rho}=\infty$, and the maximum values of the enhanced settling velocity are about $15 \%$ and $50 \%$ for $U_{e s} / u_{r . m . s .}$ and $U_{e s} / U_{s}$, respectively. Basically, it shows a decrease of about one-fifth compared with the respective enhanced settling velocities of the heavy-particle case discussed in the previous paragraph. In order to get a better view on the effect of decreasing $R_{\rho}$ we take a horizontal and vertical cut of Fig. 6, which is shown in Figs. 7(a) and 7(b), where also results with $R_{\rho}=100,2$ and 1.2 (only left panel) are included.

From Fig. 7 we can clearly see that the enhanced settling is reduced when lowering $R_{\rho}$. This is in agreement with the results for the single vortex case, where we see that the pressure gradient force keeps the particles longer inside the vortices. To investigate this in somewhat more detail, we explore the influence of the contributions from different combinations of hydrodynamic forces; see Fig. 8.

In Fig. 8 we illustrate the influence of the pressure gradient force and the Basset history force (in four combinations) on enhanced settling for $R_{\rho}=100$ and $R_{\rho}=10$. In the case where $R_{\rho}=100$ the Basset history force is much more important than the pressure gradient term (which has been reported before for nonheavy particles in isotropic turbulence; see Ref. [37]). Adding the pressure gradient force while the Basset history force was already taken into account does not change significantly the enhanced settling velocity over the full range of $\mathrm{St}^{*}$. As explained before, we expected this behavior from the equations of motion; the Basset history force scales with $\sqrt{\beta}$ and the pressure gradient force with $\beta$. Furthermore, the Basset history force can both increase and decrease the enhanced settling velocity, depending on the value of the Stokes number. For the case with $R_{\rho}=100$ [see Fig. 8(a)],
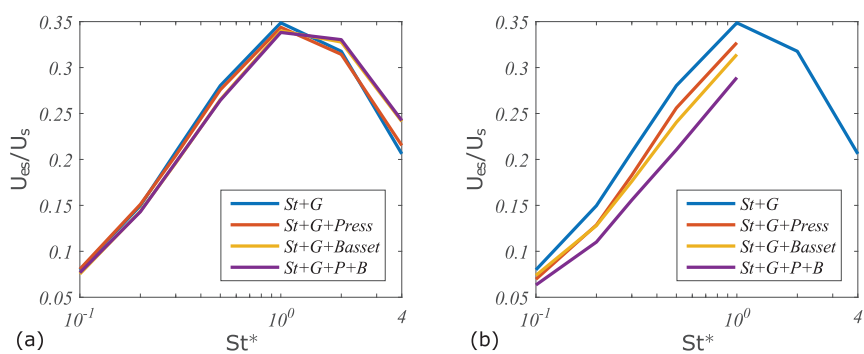

FIG. 8. Relative enhanced settling velocity is shown as a function of $\mathrm{St}^{*}$ with $\mathrm{Sv}^{*}=0.4$ (horizontal cut of Fig. 6). The different lines correspond to different combinations of hydrodynamic forces (in particular, the pressure gradient force and the Basset history force), in order to investigate the influence of these forces. Two different density ratios are used, and shown in (a) $R_{\rho}=100$ and (b) $R_{\rho}=10$.

the Basset force contributes to an increase of the enhanced settling velocity for $\mathrm{St}^{*} \gtrsim 1.5$ and to a decrease otherwise. In the numerical experiment with the single vortex we already observed that the enhanced settling velocity can be increased by the Basset history force, but the turbulence simulations clearly show that for smaller Stokes numbers a decrease is also possible. This might be due to the suppression of the inertial effects by the Basset force. Previous studies [37] have shown for almost neutrally buoyant particles in isotropic turbulence that both the Basset and the Stokes drag forces decrease the relative velocity of the particles. These results are consistent with those reported by Candelier et al. [38]. They show that just neglecting the Basset force leads to an overestimation of the ejection of nonheavy particles out of vortex cores. We expect that this particular role of the Basset force will be reflected in a slight shift to the right of the enhanced settling velocity curve (as function of the Stokes number), as shown in Fig. 8(a) when the Basset force is included.

As expected for $R_{\rho}=10$, see Fig. 8(b), the pressure gradient force becomes more important as it significantly affects enhanced settling even when added while the Basset history force was already included. The pressure gradient force decreases the enhanced settling velocity, as it forces particles toward the center of a vortex, keeping them there for a longer time.

\section{THE INFLUENCE OF THE NONLINEAR DRAG FORCE}

The Maxey-Riley equation, Eq. (4) in the form as we use it, holds provided the particle Reynolds number $\operatorname{Re}_{p}$ remains small enough, basically $\operatorname{Re}_{p} \lesssim O(1)$. For heavy particles in turbulence this is confirmed by Wang and Maxey [4] and also by Yang and Shy [39]. If the particle Reynolds number becomes of order one, nonlinear effects become important. In this section we are going to explore briefly the influence of the nonlinear drag force in order to validate the results presented in Sec. IV (i.e., to show that the relative contribution of nonlinear drag to enhanced settling is limited for the parameter regime under consideration). In order to investigate this behavior, we use a slightly modified version of the equation of motion for 

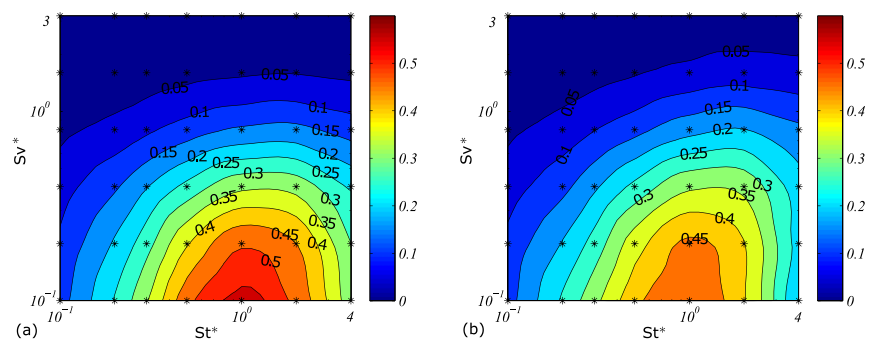

FIG. 9. Relative enhanced settling velocity $U_{e s} / U_{t}$. Both plots are taken with $R_{\rho}=100$ and as functions of $\mathrm{St}^{*}$ and $\mathrm{Sv}^{*}$. (a) Linear Stokes drag and (b) nonlinear Stokes drag. Although the differences in the relative settling velocity between the plots are small, the settling velocity itself changes more drastically, because $U_{t}$ is changed.

the particles,

$$
\begin{aligned}
\frac{d \mathbf{u}_{p}}{d t}= & f \frac{\mathbf{u}-\mathbf{u}_{p}}{\tau_{p}^{*}}+\beta \frac{D \mathbf{u}}{D t}-(1-\beta) g \mathbf{e}_{z} \\
& +\sqrt{\frac{3 \beta}{\pi \tau_{p}^{*}}} \int_{-\infty}^{t} \frac{d \mathbf{u}(\tau) / d \tau-d \mathbf{u}_{p}(\tau) / d \tau}{\sqrt{t-\tau}} d \tau,
\end{aligned}
$$

where the factor $f$ is included to account for nonlinear drag effects. Several empirical drag laws are available in the literature, among those the nonlinear drag law proposed by Schiller and Neumann [40],

$$
f=1+0.15 \operatorname{Re}_{p}^{0.687},
$$

which is appropriate for particle Reynolds numbers up to 800 [41]. We will use Eq. (16) if we refer to the case of nonlinear drag and $f=1$ in case of linear drag. Note that by introducing the nonlinear drag the terminal velocity also changes; therefore, we introduce $U_{t}$ as the actual terminal velocity (used for the relative enhanced settling velocity), while we keep the definition of $U_{s}$ the same as in Eq. (7) (used for the definition of $\mathrm{St}^{*}$ and $\mathrm{Sv}^{*}$ ). So, in case of nonlinear drag, $U_{t}$ can be calculated by solving the equation

$$
\left[1+0.15\left(\frac{2 a}{v} U_{t}\right)^{0.687}\right] U_{t}=\tau_{p}^{*}(1-\beta) g,
$$

and in the case of linear drag $U_{t}=U_{s}$ because $a \rightarrow 0$ for $f=1$. Because of this we start by investigating the case of $R_{\rho}=100$ and not $R_{\rho}=\infty$ as in the case $R_{\rho} \rightarrow \infty$ also $\frac{a}{\eta} \rightarrow 0$ [see Eq. (14)], and nonlinear drag is absent.

From Fig. 9 we can observe that the relative enhanced settling velocity has not changed much by introducing the nonlinear drag force. In absolute terms the enhanced settling velocity has decreased significantly due to the nonlinear drag force; hence, $f>1$. In Fig. 10 we show horizontal and vertical cuts of Fig. 9 (and added also results for other values of $R_{\rho}$ ). In Fig. 10(b) we observe a slight decrease of the enhanced settling velocities for small $\mathrm{Sv}^{*}$ when nonlinear effects are included. However, for high gravity, $\mathrm{Sv}^{*} \gtrsim 1$, and low density ratios, here $R_{\rho}=2$, we observe a relatively large increase in the enhanced settling velocity due to nonlinear effects. This should be considered with care and could be due to the specific definition of $\mathrm{St}^{*}$ and $\mathrm{Sv}^{*}$ (and is possibly an artifact).
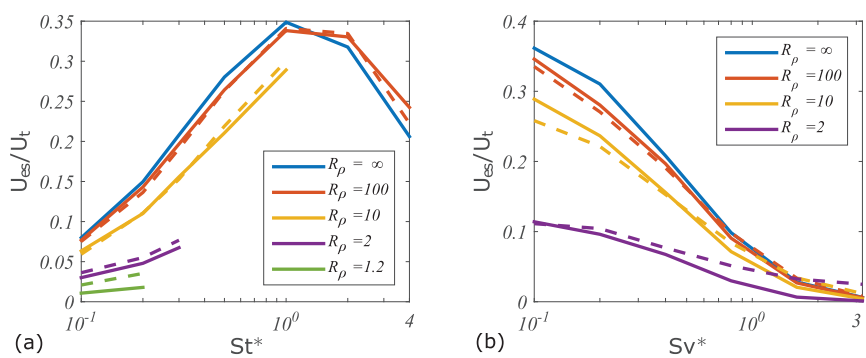

FIG. 10. Relative enhanced settling velocity for different density ratios. The solid lines are obtained excluding the nonlinear drag, while the dashed lines include the nonlinear drag. For $R_{\rho}=\infty$ the dashed and solid lines are exactly the same (here, $\frac{a}{\eta} \rightarrow 0$ resulting in the linear drag regime). (a) $U_{e s} / U_{t}$ as a function of $\mathrm{St}^{*}$ with $\mathrm{Sv}^{*}=0.4$ (horizontal cut of Fig. 9), and (b) $U_{e s} / U_{t}$ as a function of $\mathrm{Sv}^{*}$ with $\mathrm{St}^{*}=0.3$ (vertical cut of Fig. 9).

In conclusion, although the influence of the nonlinear drag on the absolute value of the settling velocity is substantial, on the relative enhanced settling velocity it is small.

\section{CONCLUSION}

We investigated the influence of different forces acting on settling particles in homogeneous and isotropic turbulence. We showed that the Maxey-Riley equation can be rewritten in a form where we have only the Stokes drag force, the pressure gradient force, gravity, and the Basset history force. The relative influence of the pressure gradient force and the Basset history force becomes more important when decreasing the particle-to-fluid density ratio $R_{\rho}$. Furthermore, the influence of the different forces on the settling velocity in the case of homogeneous isotropic turbulence is also reflected by the following observations. For $R_{\rho}=100$ the Basset history force is much more important than the pressure gradient force. The Basset history force increases the enhanced settling velocity for high Stokes numbers but decreases it for smaller Stokes numbers. Second, the pressure gradient force only decreases the settling velocity by keeping the particles longer inside the vortices, as illustrated by the single vortex case. The decrease is mostly observed for $R_{\rho}=10$ and lower. Finally, although the nonlinear drag force is important for the absolute value of the settling velocity, it is less important for the relative enhanced settling velocity for the current parameter settings.

\section{ACKNOWLEDGMENTS}

The authors gratefully acknowledge financial support from the Dutch Foundation for Fundamental Research on Matter (FOM) (Program 112 "Droplets in Turbulent Flow"). This work was sponsored by the Stichting Nationale Computerfaciliteiten (NCF) for the use of supercomputer facilities (Project No. SH-334-15), with financial support from the Netherlands Organization for Scientific Research (NWO). This article is based upon work from COST Action No. MP1305, supported by COST (European Cooperation in Science and Technology). Finally, we would like to thank Mike Machielsen for helping us by executing some early simulations in this study. 
[1] W. A. Sirignano, Fluid Dynamics and Transport of Droplets and Sprays (Cambridge University Press, Cambridge, UK, 1999).

[2] L. Metcalf and M. P. Eddy, Wastewater Engineering: Treatment and Resource Recovery (McGraw Hill, New York, 2014).

[3] F. Toschi and E. Bodenschatz, Annu. Rev. Fluid Mech. 41, 375 (2009).

[4] L.-P. Wang and M. R. Maxey, J. Fluid Mech. 256, 27 (1993).

[5] C. Y. Yang and U. Lei, J. Fluid Mech. 371, 179 (1998).

[6] J. Dávila and J. C. R. Hunt, J. Fluid Mech. 440, 117 (2001).

[7] A. Aliseda, A. Cartellier, F. Hainaux, and J. C. Lasheras, J. Fluid Mech. 468, 77 (2002).

[8] H. Parishani, O. Ayala, B. Rosa, L.-P. Wang, and W. W. Grabowski, Phys. Fluids 27, 033304 (2015).

[9] S. Elgobashi and G. C. Truesdell, J. Fluid Mech. 242, 655 (1992).

[10] V. Armenio and V. Fiorotto, Phys. Fluids 13, 2437 (2001).

[11] M. van Aartrijk and H. J. H. Clercx, Phys. Fluids 22, 013301 (2010).

[12] M. R. Maxey and J. J. Riley, Phys. Fluids 26, 883 (1983).

[13] M. A. T. van Hinsberg, J. H. M. ten Thije Boonkkamp, and H. J. H. Clercx, J. Comput. Phys. 230, 1465 (2011).

[14] A. Daitche, J. Comput. Phys. 254, 93 (2013).

[15] P. A. Moreno-Casas and F. A. Bombardelli, Environ. Fluid Mech. 16, 193 (2016).

[16] H. Faxén, Ann. Phys. 373, 89 (1922).

[17] R. Gatignol, J. Mech. Theor. Appl. 2(2), 143 (1983).

[18] J. Happel and H. Brenner, Low Reynolds Number Hydrodynamics (Kluwer, Dordrecht, 1991).

[19] E. Calzavarini, R. Volk, E. Lévêque, J.-F. Pinton, and F. Toschi, Physica D 241, 237 (2012).

[20] W. Fornari, F. Picano, and L. Brandt, J. Fluid Mech. 788, 640 (2016).

[21] W. Fornari, F. Picano, G. Sardina, and L. Brandt, J. Fluid Mech. 808, 153 (2016).
[22] S. Elgobashi, Appl. Sci. Res. 48, 301 (1991).

[23] L. Biferale, A. S. Lanotte, R. Scatamacchia, and F. Toschi, J. Fluid Mech. 757, 550 (2014).

[24] A. G. Lamorgese, D. A. Caughey, and S. B. Pope, Phys. Fluids 17, 015106 (2005).

[25] A. B. Basset, Treatise on Hydrodynamics (Deighton, Bell and Co., Cambridge, UK, 1888), Vol. 2.

[26] E. E. Michaelides, J. Fluids Eng. 125, 209 (2003).

[27] R. Mei, Exp. Fluids 22, 1 (1996).

[28] J. Magnaudet and I. Eames, Annu. Rev. Fluid Mech. 32, 659 (2000).

[29] A. Daitche and T. Tél, Phys. Rev. Lett. 107, 244501 (2011).

[30] K. Guseva, A. Daitche, U. Feudel, and T. Tél, Phys. Rev. Fluids 1, 074203 (2016).

[31] S. Olivieri, F. Picano, G. Sardina, D. Iudicone, and L. Brandt, Phys. Fluids 26, 041704 (2014).

[32] M. A. T. van Hinsberg, J. H. M. ten Thije Boonkkamp, F. Toschi, and H. J. H. Clercx, SIAM J. Sci. Comp. 34, B479 (2012).

[33] M. A. T. van Hinsberg, J. H. M. ten Thije Boonkkamp, F. Toschi, and H. J. H. Clercx, Phys. Rev. E 87, 043307 (2013).

[34] P. J. Ireland, T. Vaithianathan, P. S. Sukheswalla, B. Ray, and L. R. Collins, Comput. Fluids 76, 170 (2013).

[35] A. J. Dorgan and E. Loth, Int. J. Multiphase Flow 33, 833 (2007).

[36] F. A. Bombardelli, A. E. Gonzáles, and Y. I. Niño, J. Hydraul. Eng. 134, 1513 (2008).

[37] M. van Aartrijk and H. J. H. Clercx, J. Hydro-environ. Res. 4, 103 (2010).

[38] F. Candelier, J. R. Angilella, and M. Souhar, Phys. Fluids 16, 1765 (2004).

[39] T. S. Yang and S. S. Shy, Phys. Fluids 15, 868 (2003).

[40] R. Clift, J. R. Grace, and M. E. Weber, Bubbles, Drops and Particles (Academic Press, New York, 1978).

[41] C. T. Crowe, M. Sommerfeld, and Y. Tsuji, Multiphase Flows with Droplets and Particles (CRC Press, Taylor \& Francis Group, Boca Raton, FL, 1998). 